\title{
On Higher-Order Sequential Fractional Differential Inclusions with Nonlocal Three-Point Boundary Conditions
}

\author{
Bashir Ahmad ${ }^{1}$ and Sotiris K. Ntouyas ${ }^{2}$ \\ ${ }^{1}$ Nonlinear Analysis and Applied Mathematics (NAAM) Research Group, Department of Mathematics, Faculty of Science, \\ King Abdulaziz University, P.O. Box 80203, Jeddah 21589, Saudi Arabia \\ ${ }^{2}$ Department of Mathematics, University of Ioannina, 45110 Ioannina, Greece
}

Correspondence should be addressed to Bashir Ahmad; bashirahmad_qau@yahoo.com

Received 18 May 2014; Accepted 27 June 2014; Published 3 August 2014

Academic Editor: Hari M. Srivastava

Copyright (c) 2014 B. Ahmad and S. K. Ntouyas. This is an open access article distributed under the Creative Commons Attribution License, which permits unrestricted use, distribution, and reproduction in any medium, provided the original work is properly cited.

We study a nonlinear three-point boundary value problem of sequential fractional differential inclusions of order $\xi+1$ with $n-1<$ $\xi \leq n, n \geq 2$. Some new existence results for convex as well as nonconvex multivalued maps are obtained by using standard fixed point theorems. The paper concludes with an example.

\section{Introduction}

The topic of fractional differential equations has attracted a great attention in the recent years. It is mainly due to the intensive development of the theory and applications of fractional calculus. In fact, the tools of fractional calculus have considerably improved the modeling of several real world phenomena in physics, chemistry, bioengineering, etc. The systematic development of theory, methods, and applications of fractional differential equations can be found in [1-6]. For some recent results on fractional differential equations and inclusions, see [7-23] and the references cited therein.

In this paper, we study the following boundary value problem:

$$
\begin{gathered}
{ }^{c} D^{\xi}(D+\lambda) x(t) \in F(t, x(t)), \\
0<t<1, \quad n-1<\xi \leq n, \\
x(0)=0, \quad x^{\prime}(0)=0, \\
x^{\prime \prime}(0)=0, \ldots, x^{(n-1)}(0)=0, \quad x(1)=\alpha x(\sigma),
\end{gathered}
$$

where ${ }^{c} D$ is the Caputo fractional derivative, $D$ is the ordinary derivative, $F:[0,1] \times \mathbb{R} \rightarrow \mathscr{P}(\mathbb{R})$ is a multivalued map, $\mathscr{P}(\mathbb{R})$ is the family of all subsets of $\mathbb{R}, 0<\sigma<1, \lambda$ is a positive real number, and $\alpha$ is a real number.

The present work is motivated by a recent paper of the authors [14], where the problem (1) was considered for a single-valued case. The existence of solutions for the given multivalued problem is discussed for three cases: (a) convexvalued maps; (b) not necessarily convex-valued maps; (c) nonconvex-valued maps. To establish the existence results, we make use of nonlinear alternative for Kakutani maps, nonlinear alternative of Leray-Schauder type for singlevalued maps, selection theorem due to Bressan and Colombo for lower semicontinuous multivalued maps with nonempty closed and decomposable values, and a fixed point theorem for contractive multivalued maps due to Covitz and Nadler. The tools employed in this paper are standard; however, their exposition in the framework of the problem at hand is new.

The paper is organized as follows: in Section 2 we recall some preliminary facts that we used in the sequel. Section 3 contains the main results and an example. In Section 4, we summarize the work obtained in this paper and discuss some special cases. 


\section{Preliminaries}

Let us recall some basic definitions of fractional calculus [2, $4,6]$.

Definition 1. For $(n-1)$-times absolutely continuous function $g:[0, \infty) \rightarrow \mathbb{R}$, the Caputo derivative of fractional order $q$ is defined as

$$
\begin{array}{r}
{ }^{c} D^{q} g(t)=\frac{1}{\Gamma(n-q)} \int_{0}^{t}(t-s)^{n-q-1} g^{(n)}(s) d s, \\
n-1<q<n, \quad n=[q]+1,
\end{array}
$$

where $[q]$ denotes the integer part of the real number $q$.

Definition 2. The Riemann-Liouville fractional integral of order $q$ is defined as

$$
I^{q} g(t)=\frac{1}{\Gamma(q)} \int_{0}^{t} \frac{g(s)}{(t-s)^{1-q}} d s, \quad q>0,
$$

provided the integral exists.

Definition 3. A function $x \in A C^{n-1}([0,1], \mathbb{R})$ is called a solution of problem $(1)$ if there exists a function $v \in L^{1}([0,1], \mathbb{R})$ with $v(t) \in F(t, x(t))$, a.e. $[0,1]$, such that ${ }^{c} D^{\xi}(D+\lambda) x(t)=$ $v(t)$, a.e. $[0,1]$, and $x(0)=0, x^{\prime}(0)=0, x^{\prime \prime}(0)=$ $0, \ldots, x^{(n-1)}(0)=0$, and $x(1)=\alpha x(\sigma)$.

For the forthcoming analysis, we define

$$
\begin{aligned}
P(t)= & P_{o}(t) \\
= & \frac{t^{n-1}}{\lambda}-\frac{(n-1) t^{n-2}}{\lambda^{2}}+\frac{(n-1)(n-2) t^{n-3}}{\lambda^{3}} \\
& -\cdots-\frac{(n-1) ! t}{\lambda^{n-1}}+\frac{(n-1) !}{\lambda^{n}}\left(1-e^{-\lambda t}\right),
\end{aligned}
$$

$n$ is odd,

$$
\begin{aligned}
P(t)= & P_{e}(t) \\
= & \frac{t^{n-1}}{\lambda}-\frac{(n-1) t^{n-2}}{\lambda^{2}}+\frac{(n-1)(n-2) t^{n-3}}{\lambda^{3}} \\
& -\cdots+\frac{(n-1) ! t}{\lambda^{n-1}}-\frac{(n-1) !}{\lambda^{n}}\left(1-e^{-\lambda t}\right),
\end{aligned}
$$

$n$ is even.

Furthermore, we assume the nonresonance condition, that is, for $P=P_{o}$ and $P=P_{e}$, we choose $\alpha$ such that

$$
P(1)-\alpha P(\sigma) \neq 0, \quad \text { for } 0<\sigma<1 .
$$

Lemma 4 (see [14]). Assume that the nonresonance condition (6) holds. Given $y \in C([0,1], \mathbb{R})$, the unique solution of the problem

$$
\begin{gathered}
{ }^{c} D^{\xi}(D+\lambda) x(t)=y(t), \quad 0<t<1, \\
x(0)=0, \quad x^{\prime}(0)=0, \\
x^{\prime \prime}(0)=0, \ldots, x^{(n-1)}(0)=0, \quad x(1)=\alpha x(\sigma)
\end{gathered}
$$

is given by

$$
\begin{aligned}
x(t)= & \int_{0}^{t} e^{-\lambda(t-s)}\left(\int_{0}^{s} \frac{(s-u)^{\xi-1}}{\Gamma(\xi)} y(u) d u\right) d s \\
& +\frac{P(t)}{P(1)-\alpha P(\sigma)} \\
& \times\left[\alpha \int_{0}^{\sigma} e^{-\lambda(\sigma-s)}\left(\int_{0}^{s} \frac{(s-u)^{\xi-1}}{\Gamma(\xi)} y(u) d u\right) d s\right. \\
& \left.\quad-\int_{0}^{1} e^{-\lambda(1-s)}\left(\int_{0}^{s} \frac{(s-u)^{\xi-1}}{\Gamma(\xi)} y(u) d u\right) d s\right],
\end{aligned}
$$

where $P(t)=P_{o}(t)$ and $P(t)=P_{e}(t)$ are given by (4) and (5), respectively.

\section{Existence Results}

We begin this section with some preliminary material on multivalued maps $[24,25]$ that we need in the sequel.

Let $C([0,1])$ denote a Banach space of continuous functions from $[0,1]$ into $\mathbb{R}$ with the norm $\|x\|=\sup _{t \in[0,1]}|x(t)|$. Let $L^{1}([0,1], \mathbb{R})$ be the Banach space of measurable functions $x:[0,1] \rightarrow \mathbb{R}$ which are Lebesgue integrable and normed by $\|x\|_{L^{1}}=\int_{0}^{1}|x(t)| d t$.

Let $(X,\|\cdot\|)$ denote a normed space. Then we define

$$
\begin{gathered}
\mathscr{P}_{\mathrm{cl}}(X)=\{\mathscr{Z} \in \mathscr{P}(X): \mathscr{Z} \text { is closed }\}, \\
\mathscr{P}_{b}(X)=\{\mathscr{Z} \in \mathscr{P}(X): \mathscr{Z} \text { is bounded }\}, \\
\mathscr{P}_{\mathrm{cp}}(X)=\{\mathscr{Z} \in \mathscr{P}(X): \mathscr{Z} \text { is compact }\}, \\
\mathscr{P}_{\mathrm{cp}, \mathrm{c}}(X)=\{\mathscr{Z} \in \mathscr{P}(X): \mathscr{Z} \text { is compact and convex }\} .
\end{gathered}
$$

Definition 5. A multivalued map $\mathfrak{G}: X \rightarrow \mathscr{P}(X)$ is convex(closed-) valued if $\mathfrak{G}(x)$ is convex (closed) for all $x \in X$.

Definition 6. The map $\mathfrak{G}$ is bounded on bounded sets if $\mathfrak{G}(\mathbb{B})=\cup_{x \in \mathbb{B}} \mathfrak{G}(x)$ is bounded in $X$ for all $\mathbb{B} \in \mathscr{P}_{b}(X)$ (i.e., $\left.\sup _{x \in \mathbb{B}}\{\sup \{|y|: y \in \mathfrak{G}(x)\}\}<\infty\right)$.

Definition 7. $\mathfrak{G}$ is called upper semicontinuous (u.s.c.) on $X$ if for each $x_{0} \in X$, the set $\mathfrak{G}\left(x_{0}\right)$ is a nonempty closed subset of $X$, and if, for each open set $N$ of $X$ containing $\mathfrak{G}\left(x_{0}\right)$, there exists an open neighborhood $\mathcal{N}_{0}$ of $x_{0}$ such that $\mathfrak{G}\left(\mathcal{N}_{0}\right) \subseteq N$.

Definition 8. $\mathfrak{G}$ is said to be completely continuous if $\mathfrak{G}(\mathbb{B})$ is relatively compact for every $\mathbb{B} \in \mathscr{P}_{b}(X)$.

If the multivalued map $\mathfrak{G}$ is completely continuous with nonempty compact values, then $\mathbb{G}$ is u.s.c. if and only if $\mathfrak{G}$ has a closed graph; that is, $x_{n} \rightarrow x_{*}, y_{n} \rightarrow y_{*}$, and $y_{n} \in \mathfrak{G}\left(x_{n}\right)$ imply that $y_{*} \in \mathfrak{G}\left(x_{*}\right)$. $\mathfrak{G}$ has a fixed point if there is $x \in X$ such that $x \in \mathfrak{G}(x)$. The fixed point set of the multivalued operator $\mathfrak{G}$ will be denoted by Fix $\mathfrak{G}$. 
Definition 9. A multivalued map $\left(\mathfrak{G}:[0 ; 1] \rightarrow \mathscr{P}_{\mathrm{cl}}(\mathbb{R})\right.$ is said to be measurable if for every $y \in \mathbb{R}$, the function

$$
t \longmapsto d(y, \mathfrak{G}(t))=\inf \{|y-z|: z \in \mathfrak{G}(t)\}
$$

is measurable.

\subsection{The Carathéodory Case}

Definition 10. A multivalued map $F:[0,1] \times \mathbb{R} \rightarrow \mathscr{P}(\mathbb{R})$ is said to be Carathéodory if

(i) $t \mapsto F(t, x)$ is measurable for each $x \in \mathbb{R}$;

(ii) $x \mapsto F(t, x)$ is upper semicontinuous for almost all $t \in[0,1]$.

Further a Carathéodory function $F$ is called $L^{1}$-Carathéodory if

(iii) for each $\rho>0$, there exists $\varphi_{\rho} \in L^{1}\left([0,1], \mathbb{R}^{+}\right)$such that

$$
\|F(t, x)\|=\sup \{|v|: v \in F(t, x)\} \leq \varphi_{\rho}(t)
$$

for all $\|x\| \leq \rho$ and for a.e. $t \in[0,1]$. by

For each $y \in C([0,1], \mathbb{R})$, define the set of selections of $F$

$$
\begin{aligned}
S_{F, y}:= & \left\{v \in L^{1}([0,1], R): v(t) \in F(t, y(t))\right. \\
& \text { for a.e. } t \in[0,1]\} .
\end{aligned}
$$

For the forthcoming analysis, we need the following lemmas.

Lemma 11 (nonlinear alternative for Kakutanimaps [26]). Let $E$ be a Banach space, $C$ a closed convex subset of $E, U$ an open subset of $C$, and $0 \in U$. Suppose that $F: \bar{U} \rightarrow \mathscr{P}_{c p, c}(C)$ is an upper semicontinuous compact map. Then either

(i) F has a fixed point in $\bar{U}$, or

(ii) there is $a u \in \partial U$ and $\lambda \in(0,1)$ with $u \in \lambda F(u)$.

Lemma 12 (see [27]). Let $X$ be a Banach space. Let $F:[0,1] \times$ $\mathbb{R} \rightarrow \mathscr{P}_{c p, c}(X)$ be an $L^{1}$-Carathéodory multivalued map and let $\Theta$ be a linear continuous mapping from $L^{1}([0,1], X)$ to $C([0,1], X)$. Then the operator

$$
\begin{aligned}
\Theta \circ S_{F}: C([0,1], X) \longrightarrow \mathscr{P}_{c p, c}(C([0,1], X)), \\
x \longmapsto\left(\Theta \circ S_{F}\right)(x)=\Theta\left(S_{F, x}\right)
\end{aligned}
$$

is a closed graph operator in $C([0,1], X) \times C([0,1], X)$.

Now we are in a position to prove the existence of the solutions for the boundary value problem (1) when the righthand side is convex-valued.
Theorem 13. Assume that the nonresonance condition (6) holds. In addition, we suppose that

$\left(H_{1}\right) F:[0,1] \times \mathbb{R} \rightarrow \mathscr{P}(\mathbb{R})$ is Carathéodory and has nonempty compact and convex values;

$\left(\mathrm{H}_{2}\right)$ there exist a continuous nondecreasing function $\psi$ : $[0, \infty) \rightarrow(0, \infty)$ and a function $p \in L^{1}\left([0,1], \mathbb{R}^{+}\right)$ such that

$$
\begin{aligned}
\|F(t, x)\|_{\mathscr{P}} & :=\sup \{|y|: y \in F(t, x)\} \\
& \leq p(t) \psi(\|x\|) \quad \text { for each }(t, x) \in[0,1] \times \mathbb{R} ;
\end{aligned}
$$

$\left(H_{3}\right)$ there exists a constant $M>0$ such that

$$
\begin{aligned}
M\left(\frac{\psi(M)}{\Gamma(\xi)}\{\right. & \left(1+P_{1}\right) \int_{0}^{1} e^{-\lambda(1-s)} p(s) d s \\
& \left.\left.+\alpha P_{1} \int_{0}^{\sigma} e^{-\lambda(\sigma-s)} p(s) d s\right\}\right)^{-1}>1,
\end{aligned}
$$

where $P_{1}=\max _{t \in[0,1]}|P(t) /(P(1)-\alpha P(\sigma))|(P(t)$ is defined in (4) and (5)).

Then the boundary value problem (1) has at least one solution on $[0,1]$.

Proof. Define the operator $\Omega_{F} \quad: \quad C([0,1], \mathbb{R}) \quad \rightarrow$ $\mathscr{P}(C([0,1], \mathbb{R}))$ by

$$
\begin{aligned}
& \Omega_{F}(x) \\
& =\{h \in C([0,1], \mathbb{R}): h(t) \\
& =\left\{\int_{0}^{t} e^{-\lambda(t-s)}\left(\int_{0}^{s} \frac{(s-u)^{\xi-1}}{\Gamma(\xi)} v(u) d u\right) d s\right. \\
& +\frac{P(t)}{P(1)-\alpha P(\sigma)} \\
& \times\left[\alpha \int_{0}^{\sigma} e^{-\lambda(\sigma-s)}\left(\int_{0}^{s} \frac{(s-u)^{\xi-1}}{\Gamma(\xi)} v(u) d u\right) d s\right. \\
& \left.\left.\quad-\int_{0}^{1} e^{-\lambda(1-s)}\left(\int_{0}^{s} \frac{(s-u)^{\xi-1}}{\Gamma(\xi)} v(u) d u\right) d s\right]\right\},
\end{aligned}
$$

for $v \in S_{F, x}$. We will show that $\Omega_{F}$ satisfies the assumptions of the nonlinear alternative of Leray-Schauder type. The proof consists of several steps. As a first step, we show that $\Omega_{F}$ is convex for each $x \in C([0,1], \mathbb{R})$. This step is obvious since $S_{F, x}$ is convex ( $F$ has convex values), and therefore we omit the proof. 
In the second step, we show that $\Omega_{F}$ maps bounded sets (balls) into bounded sets in $C([0,1], \mathbb{R})$. For a positive number $r$, let $B_{r}=\{x \in C([0,1], \mathbb{R}):\|x\| \leq r\}$ be a bounded ball in $C([0,1], \mathbb{R})$. Then, for each $h \in \Omega_{F}(x), x \in B_{r}$, there exists $v \in S_{F, x}$ such that

$$
\begin{aligned}
h(t)= & \int_{0}^{t} e^{-\lambda(t-s)}\left(\int_{0}^{s} \frac{(s-u)^{\xi-1}}{\Gamma(\xi)} v(u) d u\right) d s \\
& +\frac{P(t)}{P(1)-\alpha P(\sigma)} \\
& \times\left[\alpha \int_{0}^{\sigma} e^{-\lambda(\sigma-s)}\left(\int_{0}^{s} \frac{(s-u)^{\xi-1}}{\Gamma(\xi)} v(u) d u\right) d s\right. \\
& \left.\quad-\int_{0}^{1} e^{-\lambda(1-s)}\left(\int_{0}^{s} \frac{(s-u)^{\xi-1}}{\Gamma(\xi)} v(u) d u\right) d s\right] .
\end{aligned}
$$

Then for $t \in[0,1]$, we have

$$
\begin{aligned}
& |h(t)| \\
& \leq \mid \int_{0}^{t} e^{-\lambda(t-s)}\left(\int_{0}^{s} \frac{(s-u)^{\xi-1}}{\Gamma(\xi)} v(u) d u\right) d s \\
& +\frac{P(t)}{P(1)-\alpha P(\sigma)} \\
& \times\left[\alpha \int_{0}^{\sigma} e^{-\lambda(\sigma-s)}\left(\int_{0}^{s} \frac{(s-u)^{\xi-1}}{\Gamma(\xi)} v(u) d u\right) d s\right. \\
& \left.+\int_{0}^{1} e^{-\lambda(1-s)}\left(\int_{0}^{s} \frac{(s-u)^{\xi-1}}{\Gamma(\xi)} v(u) d u\right) d s\right] \mid \\
& \leq \int_{0}^{t} e^{-\lambda(t-s)}\left(\int_{0}^{s} \frac{(s-u)^{\xi-1}}{\Gamma(\xi)} p(s) \psi(\|x\|) d u\right) d s \\
& +\left|\frac{P(t)}{P(1)-\alpha P(\sigma)}\right| \\
& \times\left[\alpha \int_{0}^{\sigma} e^{-\lambda(\sigma-s)}\left(\int_{0}^{s} \frac{(s-u)^{\xi-1}}{\Gamma(\xi)} p(s) \psi(\|x\|) d u\right) d s\right. \\
& \left.+\int_{0}^{1} e^{-\lambda(1-s)}\left(\int_{0}^{s} \frac{(s-u)^{\xi-1}}{\Gamma(\xi)} p(s) \psi(\|x\|) d u\right) d s\right] \\
& \leq \frac{\psi(\|x\|)}{\Gamma(\xi)}\left\{\int_{0}^{1} e^{-\lambda(1-s)} p(s) d s+\alpha P_{1} \int_{0}^{\sigma} e^{-\lambda(\sigma-s)} p(s) d s\right. \\
& \left.+P_{1} \int_{0}^{1} e^{-\lambda(1-s)} p(s) d s\right\} \\
& =\frac{\psi(\|x\|)}{\Gamma(\xi)}\left\{\left(1+P_{1}\right) \int_{0}^{1} e^{-\lambda(1-s)} p(s) d s\right. \\
& \left.+\alpha P_{1} \int_{0}^{\sigma} e^{-\lambda(\sigma-s)} p(s) d s\right\} .
\end{aligned}
$$

Consequently,

$$
\begin{array}{r}
\|h\| \leq \frac{\psi(r)}{\Gamma(\xi)}\left\{\left(1+P_{1}\right) \int_{0}^{1} e^{-\lambda(1-s)} p(s) d s\right. \\
\left.+\alpha P_{1} \int_{0}^{\sigma} e^{-\lambda(\sigma-s)} p(s) d s\right\} .
\end{array}
$$

Now we show that $\Omega_{F}$ maps bounded sets into equicontinuous sets of $C([0,1], \mathbb{R})$. Let $t_{1}, t_{2} \in[0,1]$ and $x \in B_{r}$. For each $h \in \Omega_{F}(x)$, we obtain

$$
\begin{aligned}
& \left|h\left(t_{1}\right)-h\left(t_{2}\right)\right| \\
& =\mid \int_{0}^{t_{1}} e^{-\lambda\left(t_{1}-s\right)}\left(\int_{0}^{s} \frac{(s-u)^{\xi-1}}{\Gamma(\xi)} v(u) d u\right) d s \\
& -\int_{0}^{t_{2}} e^{-\lambda\left(t_{2}-s\right)}\left(\int_{0}^{s} \frac{(s-u)^{\xi-1}}{\Gamma(\xi)} v(u) d u\right) d s \\
& +\frac{\left[P\left(t_{1}\right)-P\left(t_{2}\right)\right]}{P(1)-\alpha P(\sigma)} \\
& \times\left[\alpha \int_{0}^{\sigma} e^{-\lambda(\sigma-s)}\left(\int_{0}^{s} \frac{(s-u)^{\xi-1}}{\Gamma(\xi)} v(u) d u\right) d s\right. \\
& \left.-\int_{0}^{1} e^{-\lambda(1-s)}\left(\int_{0}^{s} \frac{(s-u)^{\xi-1}}{\Gamma(\xi)} v(u) d u\right) d s\right] \mid \\
& \leq \int_{0}^{t_{1}} e^{-\lambda\left(t_{1}-s\right)}\left(\int_{0}^{s} \frac{(s-u)^{\xi-1}}{\Gamma(\xi)} \psi(r) p(u) d u\right) d s \\
& -\int_{0}^{t_{2}} e^{-\lambda\left(t_{2}-s\right)}\left(\int_{0}^{s} \frac{(s-u)^{\xi-1}}{\Gamma(\xi)} \psi(r) p(u) d u\right) d s \\
& +\left|\frac{P\left(t_{1}\right)-P\left(t_{2}\right)}{P(1)-\alpha P(\sigma)}\right| \\
& \times\left[\alpha \int_{0}^{\sigma} e^{-\lambda(\sigma-s)}\left(\int_{0}^{s} \frac{(s-u)^{\xi-1}}{\Gamma(\xi)} \psi(r) p(u) d u\right) d s\right. \\
& \left.-\int_{0}^{1} e^{-\lambda(1-s)}\left(\int_{0}^{s} \frac{(s-u)^{\xi-1}}{\Gamma(\xi)} \psi(r) p(u) d u\right) d s\right] .
\end{aligned}
$$

Obviously the right-hand side of the above inequality tends to zero independently of $x \in B_{r}$ as $t_{2}-t_{1} \rightarrow 0$. As $\Omega_{F}$ satisfies the above three assumptions, therefore it follows from the Ascoli-Arzelá theorem that $\Omega_{F}: C([0,1], \mathbb{R}) \rightarrow$ $\mathscr{P}(C([0,1], \mathbb{R}))$ is completely continuous. 
In our next step, we show that $\Omega_{F}$ has a closed graph. Let $x_{n} \rightarrow x_{*}, h_{n} \in \Omega_{F}\left(x_{n}\right)$, and $h_{n} \rightarrow h_{*}$. Then we need to show that $h_{*} \in \Omega_{F}\left(x_{*}\right)$. Associated with $h_{n} \in \Omega_{F}\left(x_{n}\right)$, there exists $v_{n} \in S_{F, x_{n}}$ such that, for each $t \in[0,1]$,

$$
\begin{aligned}
h_{n}(t)= & \int_{0}^{t} e^{-\lambda(t-s)}\left(\int_{0}^{s} \frac{(s-u)^{\xi-1}}{\Gamma(\xi)} v_{n}(u) d u\right) d s \\
& +\frac{P(t)}{P(1)-\alpha P(\sigma)} \\
& \times\left[\alpha \int_{0}^{\sigma} e^{-\lambda(\sigma-s)}\left(\int_{0}^{s} \frac{(s-u)^{\xi-1}}{\Gamma(\xi)} v_{n}(u) d u\right) d s\right. \\
& \left.\quad-\int_{0}^{1} e^{-\lambda(1-s)}\left(\int_{0}^{s} \frac{(s-u)^{\xi-1}}{\Gamma(\xi)} v_{n}(u) d u\right) d s\right] .
\end{aligned}
$$

Thus, it suffices to show that there exists $v_{*} \in S_{F, x_{*}}$ such that, for each $t \in[0,1]$,

$$
\begin{aligned}
h_{*}(t)= & \int_{0}^{t} e^{-\lambda(t-s)}\left(\int_{0}^{s} \frac{(s-u)^{\xi-1}}{\Gamma(\xi)} v_{*}(u) d u\right) d s \\
& +\frac{P(t)}{P(1)-\alpha P(\sigma)} \\
& \times\left[\alpha \int_{0}^{\sigma} e^{-\lambda(\sigma-s)}\left(\int_{0}^{s} \frac{(s-u)^{\xi-1}}{\Gamma(\xi)} v_{*}(u) d u\right) d s\right. \\
& \left.\quad-\int_{0}^{1} e^{-\lambda(1-s)}\left(\int_{0}^{s} \frac{(s-u)^{\xi-1}}{\Gamma(\xi)} v_{*}(u) d u\right) d s\right] .
\end{aligned}
$$

Let us consider the linear operator $\Theta: L^{1}([0,1], \mathbb{R}) \rightarrow$ $C([0,1], \mathbb{R})$ given by

$$
\begin{aligned}
f \longmapsto & \Theta(v)(t) \\
= & \int_{0}^{t} e^{-\lambda(t-s)}\left(\int_{0}^{s} \frac{(s-u)^{\xi-1}}{\Gamma(\xi)} v(u) d u\right) d s \\
& +\frac{P(t)}{P(1)-\alpha P(\sigma)} \\
& \times\left[\alpha \int_{0}^{\sigma} e^{-\lambda(\sigma-s)}\left(\int_{0}^{s} \frac{(s-u)^{\xi-1}}{\Gamma(\xi)} v(u) d u\right) d s\right. \\
& \left.\quad-\int_{0}^{1} e^{-\lambda(1-s)}\left(\int_{0}^{s} \frac{(s-u)^{\xi-1}}{\Gamma(\xi)} v(u) d u\right) d s\right] .
\end{aligned}
$$

Observe that

$$
\begin{aligned}
& \left\|h_{n}(t)-h_{*}(t)\right\| \\
& =\| \int_{0}^{t} e^{-\lambda(t-s)}\left(\int_{0}^{s} \frac{(s-u)^{\xi-1}}{\Gamma(\xi)}\left(v_{n}(u)-v_{*}(u)\right) d u\right) d s \\
& +\frac{P(t)}{P(1)-\alpha P(\sigma)} \\
& \times\left[\alpha \int_{0}^{\sigma} e^{-\lambda(\sigma-s)}\left(\int_{0}^{s} \frac{(s-u)^{\xi-1}}{\Gamma(\xi)}\left(v_{n}(u)-v_{*}(u)\right) d u\right) d s\right. \\
& \left.\quad-\int_{0}^{1} e^{-\lambda(1-s)}\left(\int_{0}^{s} \frac{(s-u)^{\xi-1}}{\Gamma(\xi)}\left(v_{n}(u)-v_{*}(u)\right) d u\right) d s\right] \| \\
& \longrightarrow 0
\end{aligned}
$$

as $n \rightarrow \infty$.

Thus, it follows from Lemma 12 that $\Theta \circ S_{F}$ is a closed graph operator. Further, we have $h_{n}(t) \in \Theta\left(S_{F, x_{n}}\right)$. Since $x_{n} \rightarrow x_{*}$, therefore, we have

$$
\begin{aligned}
h_{*}(t)= & \int_{0}^{t} e^{-\lambda(t-s)}\left(\int_{0}^{s} \frac{(s-u)^{\xi-1}}{\Gamma(\xi)} v_{*}(u) d u\right) d s \\
& +\frac{P(t)}{P(1)-\alpha P(\sigma)} \\
& \times\left[\alpha \int_{0}^{\sigma} e^{-\lambda(\sigma-s)}\left(\int_{0}^{s} \frac{(s-u)^{\xi-1}}{\Gamma(\xi)} v_{*}(u) d u\right) d s\right. \\
& \left.\quad-\int_{0}^{1} e^{-\lambda(1-s)}\left(\int_{0}^{s} \frac{(s-u)^{\xi-1}}{\Gamma(\xi)} v_{*}(u) d u\right) d s\right],
\end{aligned}
$$

for some $v_{*} \in S_{F, x_{*}}$.

Finally, we show that there exists an open set $U \subseteq$ $C([0,1], \mathbb{R})$ with $x \notin \Omega_{F}(x)$ for any $\lambda \in(0,1)$ and all $x \in \partial U$. Let $\lambda \in(0,1)$ and $x \in \lambda \Omega_{F}(x)$. Then there exists $v \in L^{1}([0,1], \mathbb{R})$ with $v \in S_{F, x}$ such that, for $t \in[0,1]$, we have

$$
\begin{aligned}
x(t)= & \int_{0}^{t} e^{-\lambda(t-s)}\left(\int_{0}^{s} \frac{(s-u)^{\xi-1}}{\Gamma(\xi)} v(u) d u\right) d s \\
& +\frac{P(t)}{P(1)-\alpha P(\sigma)} \\
& \times\left[\alpha \int_{0}^{\sigma} e^{-\lambda(\sigma-s)}\left(\int_{0}^{s} \frac{(s-u)^{\xi-1}}{\Gamma(\xi)} v(u) d u\right) d s\right. \\
& \left.\quad-\int_{0}^{1} e^{-\lambda(1-s)}\left(\int_{0}^{s} \frac{(s-u)^{\xi-1}}{\Gamma(\xi)} v(u) d u\right) d s\right] .
\end{aligned}
$$


Using the computations of the second step above we have

$$
\begin{aligned}
|x(t)| \leq & \frac{\psi(\|x\|)}{\Gamma(\xi)} \\
& \times\left\{\left(1+P_{1}\right) \int_{0}^{1} e^{-\lambda(1-s)} p(s) d s\right. \\
& \left.+\alpha P_{1} \int_{0}^{\sigma} e^{-\lambda(\sigma-s)} p(s) d s\right\} .
\end{aligned}
$$

Consequently, we have

$$
\begin{aligned}
\|x\|\left(\frac{\psi(\|x\|)}{\Gamma(\xi)}\{\right. & \left(1+P_{1}\right) \int_{0}^{1} e^{-\lambda(1-s)} p(s) d s \\
& \left.\left.+\alpha P_{1} \int_{0}^{\sigma} e^{-\lambda(\sigma-s)} p(s) d s\right\}\right)^{-1} \leq 1 .
\end{aligned}
$$

In view of $\left(H_{3}\right)$, there exists $M$ such that $\|x\| \neq M$. Let us set

$$
U=\{x \in C([0,1], \mathbb{R}):\|x\|<M\} .
$$

Note that the operator $\Omega_{F}: \bar{U} \rightarrow \mathscr{P}(C([0,1], \mathbb{R}))$ is upper semicontinuous and completely continuous. From the choice of $U$, there is no $x \in \partial U$ such that $x \in \lambda \Omega_{F}(x)$ for some $\lambda \in(0,1)$. Consequently, by the nonlinear alternative of Leray-Schauder type (Lemma 11), we deduce that $\Omega_{F}$ has a fixed point $x \in \bar{U}$ which is a solution of the problem (1). This completes the proof.

Remark 14. The condition $\left(\mathrm{H}_{3}\right)$ in the statement of Theorem 13 may be replaced with the following one.

$\left(H_{3}\right)^{\prime}$ There exists a constant $M>0$ such that

$$
\frac{M}{(\psi(M) / \Gamma(\xi))\left\{\left(1+(1+\alpha) P_{1}\right)\|p\|_{L^{1}}\right\}}>1,
$$

where $P_{1}$ is the same as defined in $\left(H_{3}\right)$.

3.2. The Lower Semicontinuous Case. As a next result, we study the case when $F$ is not necessarily convex-valued. Our strategy to deal with this problem is based on the nonlinear alternative of Leray-Schauder type together with the selection theorem of Bressan and Colombo [28] for lower semicontinuous maps with decomposable values.

Let $X$ be a nonempty closed subset of a Banach space $E$ and let $G: X \rightarrow \mathscr{P}(E)$ be a multivalued operator with nonempty closed values. $G$ is lower semicontinuous (l.s.c.) if the set $\{y \in X: G(y) \cap B \neq \emptyset\}$ is open for any open set $B$ in $E$. Let $A$ be a subset of $[0,1] \times \mathbb{R}$. $A$ is $\mathscr{L} \otimes \mathscr{B}$ measurable if $A$ belongs to the $\sigma$-algebra generated by all sets of the form $\mathscr{J} \times \mathscr{D}$, where $\mathcal{J}$ is Lebesgue measurable in $[0,1]$ and $\mathscr{D}$ is Borel measurable in $\mathbb{R}$. A subset $\mathscr{A}$ of $L^{1}([0,1], \mathbb{R})$ is decomposable if, for all $u, v \in \mathscr{A}$ and measurable $\mathscr{J} \subset[0,1]=$ $J$, the function $u \chi_{\mathscr{F}}+v \chi_{J-\mathscr{J}} \in \mathscr{A}$, where $\chi_{\mathscr{F}}$ stands for the characteristic function of $\mathscr{J}$.
Definition 15. Let $Y$ be a separable metric space and let $N$ : $Y \rightarrow \mathscr{P}\left(L^{1}([0,1], \mathbb{R})\right)$ be a multivalued operator. We say $N$ has a property (BC) if $N$ is lower semicontinuous (l.s.c.) and has nonempty closed and decomposable values.

Let $F:[0,1] \times \mathbb{R} \rightarrow \mathscr{P}(\mathbb{R})$ be a multivalued map with nonempty compact values. Define a multivalued operator $\mathscr{F}$ : $C([0,1] \times \mathbb{R}) \rightarrow \mathscr{P}\left(L^{1}([0,1], \mathbb{R})\right)$ associated with $F$ as

$$
\mathscr{F}(x)=\left\{w \in L^{1}([0,1], \mathbb{R}): w(t) \in F(t, x(t))\right.
$$

$$
\text { for a.e. } t \in[0,1]\} \text {, }
$$

which is called the Nemytskii operator associated with $F$.

Definition 16. Let $F:[0,1] \times \mathbb{R} \rightarrow \mathscr{P}(\mathbb{R})$ be a multivalued function with nonempty compact values. We say $F$ is of lower semicontinuous type (l.s.c. type) if its associated Nemytskii operator $\mathscr{F}$ is lower semicontinuous and has nonempty closed and decomposable values.

Lemma 17 (see [29]). Let $Y$ be a separable metric space and let $N: Y \rightarrow \mathscr{P}\left(L^{1}([0,1], \mathbb{R})\right)$ be a multivalued operator satisfying the property $(B C)$. Then $N$ has a continuous selection; that is, there exists a continuous function (singlevalued) $g: Y \rightarrow L^{1}([0,1], \mathbb{R})$ such that $g(x) \in N(x)$ for every $x \in Y$.

Theorem 18. Assume that $\left(\mathrm{H}_{2}\right),\left(\mathrm{H}_{3}\right)$, and the following condition hold:

\section{$\left(H_{4}\right) F:[0,1] \times \mathbb{R} \rightarrow \mathscr{P}(\mathbb{R})$ is a nonempty compact-valued multivalued map such that}
(a) $(t, x) \mapsto F(t, x)$ is $\mathscr{L} \otimes \mathscr{B}$ measurable,
(b) $x \mapsto F(t, x)$ is lower semicontinuous for each $t \in$ $[0,1]$

Further the nonresonance condition (6) holds. Then the boundary value problem (1) has at least one solution on $[0,1]$.

Proof. It follows from $\left(\mathrm{H}_{2}\right)$ and $\left(\mathrm{H}_{4}\right)$ that $F$ is of l.s.c. type. Then from Lemma 17, there exists a continuous function $f$ : $A C^{1}([0,1], \mathbb{R}) \rightarrow L^{1}([0,1], \mathbb{R})$ such that $f(x) \in \mathscr{F}(x)$ for all $x \in C([0,1], \mathbb{R})$.

Consider the problem

$$
\begin{gathered}
{ }^{c} D^{\xi}(D+\lambda) x(t)=f(x(t)), \quad 0<t<1, \\
x(0)=0, \quad x^{\prime}(0)=0, \\
x^{\prime \prime}(0)=0, \ldots, x^{(n-1)}(0)=0, \quad x(1)=\alpha x(\sigma) .
\end{gathered}
$$

Observe that if $x \in A C^{1}([0,1], \mathbb{R})$ is a solution of $(32)$, then $x$ is a solution to the problem (1). In order to transform 
the problem (32) into a fixed point problem, we define the operator $\overline{\Omega_{F}}$ as

$$
\begin{aligned}
\overline{\Omega_{F}} x(t)= & \int_{0}^{t} e^{-\lambda(t-s)}\left(\int_{0}^{s} \frac{(s-u)^{\xi-1}}{\Gamma(\xi)} f(x(u)) d u\right) d s \\
& +\frac{P(t)}{P(1)-\alpha P(\sigma)} \\
& \times\left[\alpha \int_{0}^{\sigma} e^{-\lambda(\sigma-s)}\left(\int_{0}^{s} \frac{(s-u)^{\xi-1}}{\Gamma(\xi)} f(x(u)) d u\right) d s\right. \\
& \left.-\int_{0}^{1} e^{-\lambda(1-s)}\left(\int_{0}^{s} \frac{(s-u)^{\xi-1}}{\Gamma(\xi)} f(x(u)) d u\right) d s\right] .
\end{aligned}
$$

It can easily be shown that $\overline{\Omega_{F}}$ is continuous and completely continuous. The remaining part of the proof is similar to that of Theorem 13. So we omit it. This completes the proof.

3.3. The Lipschitz Case. Now we prove the existence of solutions for the problem (1) with a nonconvex-valued righthand side by applying a fixed point theorem for multivalued map due to Covitz and Nadler [30].

Let $(X, d)$ be a metric space induced from the normed space $(X,\|\cdot\|)$. Consider $H_{d}: \mathscr{P}(X) \times \mathscr{P}(X) \rightarrow \mathbb{R} \cup\{\infty\}$ given by

$$
H_{d}(A, B)=\max \left\{\sup _{a \in A} d(a, B), \sup _{b \in B} d(A, b)\right\},
$$

where $d(A, b)=\inf _{a \in A} d(a ; b)$ and $d(a, B)=\inf _{b \in B} d(a ; b)$. Then $\left(\mathscr{P}_{b, \mathrm{cl}}(X), H_{d}\right)$ is a metric space and $\left(\mathscr{P}_{\mathrm{cl}}(X), H_{d}\right)$ is a generalized metric space (see [31]).

Definition 19. A multivalued operator $N: X \rightarrow \mathscr{P}_{\mathrm{cl}}(X)$ is called

(a) $\gamma$-Lipschitz if and only if there exists $\gamma>0$ such that

$H_{d}(N(x), N(y)) \leq \gamma d(x, y) \quad$ for each $x, y \in X$

(b) a contraction if and only if it is $\gamma$-Lipschitz with $\gamma<1$.

Lemma 20 (see [30]). Let $(X, d)$ be a complete metric space. If $N: X \rightarrow \mathscr{P}_{c l}(X)$ is a contraction, then Fix $N \neq \emptyset$.

Theorem 21. Assume that the nonresonance condition (6) holds. In addition, suppose that the following conditions hold:

$\left(H_{5}\right) F:[0,1] \times \mathbb{R} \rightarrow \mathscr{P}_{c p}(\mathbb{R})$ is such that $F(\cdot, x):[0,1] \rightarrow$ $\mathscr{P}_{c p}(\mathbb{R})$ is measurable for each $x \in \mathbb{R}$;

$\left(H_{6}\right) H_{d}(F(t, x), F(t, \bar{x})) \leq m(t)|x-\bar{x}|$ for almost all $t \in$ $[0,1]$ and $x, \bar{x} \in \mathbb{R}$ with $m \in L^{1}\left([0,1], \mathbb{R}^{+}\right)$and $d(0, F(t, 0)) \leq m(t)$ for almost all $t \in[0,1]$.
Then the boundary value problem (1) has at least one solution on $[0,1]$ if

$$
\begin{aligned}
& \frac{1}{\Gamma(\xi)}\left\{\left(1+P_{1}\right) \int_{0}^{1} e^{-\lambda(1-s)} m(s) d s\right. \\
& \left.\quad+\alpha P_{1} \int_{0}^{\sigma} e^{-\lambda(\sigma-s)} m(s) d s\right\}<1 .
\end{aligned}
$$

Proof. Observe that the set $S_{F, x}$ is nonempty for each $x \in$ $C([0,1], \mathbb{R})$ by the assumption $\left(H_{5}\right)$, so $F$ has a measurable selection (see Theorem III.6 [32]). Now we show that the operator $\Omega_{F}$, defined in the beginning of proof of Theorem 13, satisfies the assumptions of Lemma 20. To show that $\Omega_{F}(x) \in$ $\mathscr{P}_{\mathrm{cl}}((C[0,1], \mathbb{R}))$ for each $x \in C([0,1], \mathbb{R})$, let $\left\{u_{n}\right\}_{n \geq 0} \in$ $\Omega_{F}(x)$ be such that $u_{n} \rightarrow u(n \rightarrow \infty)$ in $C([0,1], \mathbb{R})$. Then $u \in C([0,1], \mathbb{R})$ and there exists $v_{n} \in S_{F, x_{n}}$ such that, for each $t \in[0,1]$,

$$
\begin{aligned}
u_{n}(t)= & \int_{0}^{t} e^{-\lambda(t-s)}\left(\int_{0}^{s} \frac{(s-u)^{\xi-1}}{\Gamma(\xi)} v_{n}(u) d u\right) d s \\
& +\frac{P(t)}{P(1)-\alpha P(\sigma)} \\
& \times\left[\alpha \int_{0}^{\sigma} e^{-\lambda(\sigma-s)}\left(\int_{0}^{s} \frac{(s-u)^{\xi-1}}{\Gamma(\xi)} v_{n}(u) d u\right) d s\right. \\
& \left.\quad-\int_{0}^{1} e^{-\lambda(1-s)}\left(\int_{0}^{s} \frac{(s-u)^{\xi-1}}{\Gamma(\xi)} v_{n}(u) d u\right) d s\right] .
\end{aligned}
$$

As $F$ has compact values, we pass onto a subsequence (if necessary) to obtain that $v_{n}$ converges to $v$ in $L^{1}([0,1], \mathbb{R})$. Thus, $v \in S_{F, x}$ and, for each $t \in[0,1]$, we have

$$
\begin{aligned}
v_{n}(t) & \longrightarrow v(t) \\
= & \int_{0}^{t} e^{-\lambda(t-s)}\left(\int_{0}^{s} \frac{(s-u)^{\xi-1}}{\Gamma(\xi)} v(u) d u\right) d s \\
+ & \frac{P(t)}{P(1)-\alpha P(\sigma)} \\
\times & {\left[\alpha \int_{0}^{\sigma} e^{-\lambda(\sigma-s)}\left(\int_{0}^{s} \frac{(s-u)^{\xi-1}}{\Gamma(\xi)} v(u) d u\right) d s\right.} \\
& \left.\quad-\int_{0}^{1} e^{-\lambda(1-s)}\left(\int_{0}^{s} \frac{(s-u)^{\xi-1}}{\Gamma(\xi)} v(u) d u\right) d s\right] .
\end{aligned}
$$

Hence, $u \in \Omega(x)$.

Next we show that there exists $\delta<1$ such that

$$
\begin{aligned}
& H_{d}\left(\Omega_{F}(x), \Omega_{F}(\bar{x})\right) \\
& \quad \leq \delta\|x-\bar{x}\| \quad \text { for each } x, \bar{x} \in A C^{1}([0,1], \mathbb{R}) .
\end{aligned}
$$


Let $x, \bar{x} \in A C^{1}([0,1], \mathbb{R})$ and $h_{1} \in \Omega_{F}(x)$. Then there exists $v_{1}(t) \in F(t, x(t))$ such that, for each $t \in[0,1]$,

$$
\begin{aligned}
h_{1}(t)= & \int_{0}^{t} e^{-\lambda(t-s)}\left(\int_{0}^{s} \frac{(s-u)^{\xi-1}}{\Gamma(\xi)} v_{1}(u) d u\right) d s \\
& +\frac{P(t)}{P(1)-\alpha P(\sigma)} \\
& \times\left[\alpha \int_{0}^{\sigma} e^{-\lambda(\sigma-s)}\left(\int_{0}^{s} \frac{(s-u)^{\xi-1}}{\Gamma(\xi)} v_{1}(u) d u\right) d s\right. \\
& \left.\quad-\int_{0}^{1} e^{-\lambda(1-s)}\left(\int_{0}^{s} \frac{(s-u)^{\xi-1}}{\Gamma(\xi)} v_{1}(u) d u\right) d s\right] .
\end{aligned}
$$

By $\left(H_{6}\right)$, we have

$$
H_{d}(F(t, x), F(t, \bar{x})) \leq m(t)|x(t)-\bar{x}(t)| .
$$

So, there exists $w \in F(t, \bar{x}(t))$ such that

$$
\left|v_{1}(t)-w\right| \leq m(t)|x(t)-\bar{x}(t)|, \quad t \in[0,1] .
$$

Define $U:[0,1] \rightarrow \mathscr{P}(\mathbb{R})$ by

$$
U(t)=\left\{w \in \mathbb{R}:\left|v_{1}(t)-w\right| \leq m(t)|x(t)-\bar{x}(t)|\right\} .
$$

Since the multivalued operator $U(t) \cap F(t, \bar{x}(t))$ is measurable (Proposition III.4 [32]), there exists a function $v_{2}(t)$ which is a measurable selection for $U$. So $v_{2}(t) \in F(t, \bar{x}(t))$ and for each $t \in[0,1]$, we have $\left|v_{1}(t)-v_{2}(t)\right| \leq m(t)|x(t)-\bar{x}(t)|$.

For each $t \in[0,1]$, let us define

$$
\begin{aligned}
h_{2}(t)= & \int_{0}^{t} e^{-\lambda(t-s)}\left(\int_{0}^{s} \frac{(s-u)^{\xi-1}}{\Gamma(\xi)} v_{2}(u) d u\right) d s \\
& +\frac{P(t)}{P(1)-\alpha P(\sigma)} \\
& \times\left[\alpha \int_{0}^{\sigma} e^{-\lambda(\sigma-s)}\left(\int_{0}^{s} \frac{(s-u)^{\xi-1}}{\Gamma(\xi)} v_{2}(u) d u\right) d s\right. \\
& \left.\quad-\int_{0}^{1} e^{-\lambda(1-s)}\left(\int_{0}^{s} \frac{(s-u)^{\xi-1}}{\Gamma(\xi)} v_{2}(u) d u\right) d s\right] .
\end{aligned}
$$

Thus,

$$
\begin{aligned}
& \left|h_{1}(t)-h_{2}(t)\right| \\
& \leq \int_{0}^{t} e^{-\lambda(t-s)}\left(\int_{0}^{s} \frac{(s-u)^{\xi-1}}{\Gamma(\xi)}\left|v_{1}(u)-v_{2}(u)\right| d u\right) d s \\
& +\left|\frac{P(t)}{P(1)-\alpha P(\sigma)}\right| \\
& \quad \times \mid \alpha \int_{0}^{\sigma} e^{-\lambda(\sigma-s)}\left(\int_{0}^{s} \frac{(s-u)^{\xi-1}}{\Gamma(\xi)}\left|v_{1}(u)-v_{2}(u)\right| d u\right) d s \\
& \left.\quad-\int_{0}^{1} e^{-\lambda(1-s)}\left(\int_{0}^{s} \frac{(s-u)^{\xi-1}}{\Gamma(\xi)}\left|v_{1}(u)-v_{2}(u)\right| d u\right) d s\right] .
\end{aligned}
$$

Hence,

$$
\begin{aligned}
& \left\|h_{1}-h_{2}\right\| \\
& \leq \frac{1}{\Gamma(\xi)}\left\{\left(1+P_{1}\right) \int_{0}^{1} e^{-\lambda(1-s)} m(s) d s\right. \\
& \left.+\alpha P_{1} \int_{0}^{\sigma} e^{-\lambda(\sigma-s)} m(s) d s\right\} \\
& \times\|x-\bar{x}\| .
\end{aligned}
$$

Analogously, interchanging the roles of $x$ and $\bar{x}$, we obtain

$$
\begin{aligned}
& H_{d}\left(\Omega_{F}(x), \Omega_{F}(\bar{x})\right) \\
& \leq \delta\|x-\bar{x}\| \\
& \leq \frac{1}{\Gamma(\xi)}\left\{\left(1+P_{1}\right) \int_{0}^{1} e^{-\lambda(1-s)} m(s) d s\right. \\
& \left.\quad+\alpha P_{1} \int_{0}^{\sigma} e^{-\lambda(\sigma-s)} m(s) d s\right\}\|x-\bar{x}\| .
\end{aligned}
$$

Since $\Omega_{F}$ is a contraction, it follows from Lemma 20 that $\Omega_{F}$ has a fixed point $x$ which is a solution of (1). This completes the proof.

Remark 22. An alternative to the condition (36) in the statement of Theorem 21 may be the following one:

$$
\frac{1}{\Gamma(\xi)}\left\{\left(1+(1+\alpha) P_{1}\right)\|m\|_{L^{1}}\right\}<1
$$

Example 23. Consider the problem

$$
\begin{gathered}
{ }^{c} D^{7 / 2}(D+2) x(t) \in F(t, x(t)), \quad 0 \leq t \leq 1, \\
x(0)=0, \quad x^{\prime}(0)=0, \\
x^{\prime \prime}(0)=0, \quad x^{\prime \prime \prime}(0)=0, \quad x(1)=x\left(\frac{1}{2}\right) .
\end{gathered}
$$

Here, $\xi=7 / 2, n=4, \lambda=2, \alpha=1, \sigma=1 / 2$, and $F$ : $[0,1] \times \mathbb{R} \rightarrow \mathscr{P}(\mathbb{R})$ is a multivalued map given by

$$
\begin{aligned}
x & \longrightarrow F(t, x) \\
& =\left[\frac{|x|^{5}}{|x|^{5}+3}+t^{3}+t^{2}+4, \frac{|x|^{3}}{|x|^{3}+1}+t+2\right] .
\end{aligned}
$$

For $f \in F$, we have

$$
|f| \leq \max \left(\frac{|x|^{5}}{|x|^{5}+3}+t^{3}+t^{2}+4, \frac{|x|^{3}}{|x|^{3}+1}+t+2\right) \leq 9
$$
$x \in \mathbb{R}$. 
Thus,

$$
\begin{aligned}
\|F(t, x)\|_{\mathscr{P}} & :=\sup \{|y|: y \in F(t, x)\} \leq 7 \\
& =p(t) \psi(\|x\|), \quad x \in \mathbb{R},
\end{aligned}
$$

with $p(t)=1, \psi(\|x\|)=7$. In this case

$$
\begin{gathered}
P(t)=P_{e}(t)=\frac{t^{3}}{2}-\frac{3 t(t-1)}{4}-\frac{3\left(1-e^{-2 t}\right)}{8}, \\
P_{1} \approx 6.214821 .
\end{gathered}
$$

By the condition $\left(\mathrm{H}_{3}\right)$, that is,

$$
\begin{aligned}
M\left(\frac { \psi ( M ) } { \Gamma ( \xi ) } \left\{\left(1+P_{1}\right) \int_{0}^{1} e^{-\lambda(1-s)} p(s) d s\right.\right. & \\
& \left.\left.+\alpha P_{1} \int_{0}^{\sigma} e^{-\lambda(\sigma-s)} p(s) d s\right\}\right)^{-1}>1,
\end{aligned}
$$

we find that $M>M_{1}$ with $M_{1} \approx 10.707326$. Therefore, it follows from Theorem 13 that problem (49) has at least one solution.

\section{Conclusions}

In this paper, we have solved a three-point boundary value problem of Caputo-type sequential fractional differential inclusions of an arbitrary order $\xi \in(n-1, n)$. The existence of solutions for the given problem with the convexvalued map is obtained by means of nonlinear alternative for Kakutani maps, while the existence result for not necessarily convex-valued map is established by combining nonlinear alternative of Leray-Schauder type for single-valued maps with a selection theorem due to Bressan and Colombo for lower semicontinuous multivalued maps with decomposable values. The nonconvex-valued case relies on a fixed point theorem for contractive multivalued maps due to Covitz and Nadler. Some new existence results follow by fixing the parameters involved in the given problem. For instance, by taking $\alpha=0$, our results correspond to a two-point Caputotype multivalued problem of an arbitrary order $\xi \in(n-1, n)$, while the results for sequential differential inclusions of order $(n+1)$ can be obtained by fixing $\xi=n$ in the results of this paper.

\section{Conflict of Interests}

The authors declare that there is no conflict of interests regarding the publication of this paper.

\section{Acknowledgments}

This paper was supported by Deanship of Scientific Research (DSR), King Abdulaziz University, Jeddah, Saudi Arabia. The authors, therefore, acknowledge technical and financial support of KAU. Sotiris K. Ntouyas is a member of Nonlinear Analysis and Applied Mathematics (NAAM) Research Group at King Abdulaziz University, Jeddah, Saudi Arabia.

\section{References}

[1] D. Baleanu, K. Diethelm, E. Scalas, and J. J. Trujillo, Fractional Calculus Models and Numerical Methods (Series on Complexity, Nonlinearity and Chaos), World Scientific, Boston, Mass, USA, 2012.

[2] A. A. Kilbas, H. M. Srivastava, and J. J. Trujillo, Theory and Applications of Fractional Differential Equations, vol. 204 of North-Holland Mathematics Studies, Elsevier Science B.V., Amsterdam, The Netherland, 2006.

[3] K. S. Miller and B. Ross, An Introduction to the Fractional Calculus and Fractional Differential Equations, John Wiley \& Sons, New York, NY, USA, 1993.

[4] I. Podlubny, Fractional Differential Equations, vol. 198, Academic Press, San Diego, Calif, USA, 1999.

[5] J. Sabatier, O. P. Agrawal, and J. A. T. Machado, Eds., Advances in Fractional Calculus: Theoretical Developments and Applications in Physics and Engineering, Springer, Dordrecht, The Netherlands, 2007.

[6] S. G. Samko, A. A. Kilbas, and O. I. Marichev, Fractional Integrals and Derivatives, Theory and Applications, Gordon and Breach Science Publishers, Yverdon, 1993.

[7] A. Aghajani, Y. Jalilian, and J. J. Trujillo, "On the existence of solutions of fractional integro-differential equations," Fractional Calculus and Applied Analysis, vol. 15, no. 1, pp. 44-69, 2012.

[8] R. P. Agarwal, B. Ahmad, A. Alsaedi, and N. Shahzad, "Existence and dimension of the set of mild solutions to semilinear fractional differential inclusions," Advances in Difference Equations, vol. 2012, article 74, 2012.

[9] B. Ahmad and S. K. Ntouyas, "Some existence results for boundary value problems of fractional differential inclusions with non-separated boundary conditions," Electronic Journal of Qualitative Theory of Differential Equations, vol. 71, pp. 1-17, 2010.

[10] B. Ahmad and S. Sivasundaram, "On four-point nonlocal boundary value problems of nonlinear integro-differential equations of fractional order," Applied Mathematics and Computation, vol. 217, no. 2, pp. 480-487, 2010.

[11] B. Ahmad and J. J. Nieto, "Existence results for a coupled system of nonlinear fractional differential equations with threepoint boundary conditions," Computers \& Mathematics with Applications, vol. 58, no. 9, pp. 1838-1843, 2009.

[12] B. Ahmad, J. J. Nieto, and A. Alsaedi, "A nonlocal three-point inclusion problem of Langevin equation with two different fractional orders," Advances in Difference Equations, vol. 2012, article 54, 16 pages, 2012.

[13] B. Ahmad and S. K. Ntouyas, "Nonlinear fractional differential equations and inclusions of arbitrary order and multi-strip boundary conditions," Electronic Journal of Differential Equations, vol. 2012, no. 98, pp. 1-22, 2012.

[14] B. Ahmad and S. K. Ntouyas, "A higher-order nonlocal threepoint boundary value problem of sequential fractional differential equations," Miskolc Mathematical Notes. To appear.

[15] Z. Bai, "On positive solutions of a nonlocal fractional boundary value problem," Nonlinear Analysis: Theory, Methods \& Applications, vol. 72, no. 2, pp. 916-924, 2010

[16] K. Balachandran and J. J. Trujillo, "The nonlocal Cauchy problem for nonlinear fractional integrodifferential equations in Banach spaces," Nonlinear Analysis: Theory, Methods \& Applications, vol. 72, no. 12, pp. 4587-4593, 2010.

[17] D. Baleanu and O. G. Mustafa, "On the global existence of solutions to a class of fractional differential equations," 
Computers \& Mathematics with Applications, vol. 59, no. 5, pp. 1835-1841, 2010.

[18] S. Bhalekar, V. Daftardar-Gejji, D. Baleanu, and R. Magin, "Fractional Bloch equation with delay," Computers \& Mathematics with Applications, vol. 61, no. 5, pp. 1355-1365, 2011.

[19] Y.-K. Chang and J. J. Nieto, "Some new existence results for fractional differential inclusions with boundary conditions," Mathematical and Computer Modelling, vol. 49, no. 3-4, pp. 605609, 2009.

[20] J. Henderson and A. Ouahab, "Fractional functional differential inclusions with finite delay," Nonlinear Analysis: Theory, Methods and Applications, vol. 70, no. 5, pp. 2091-2105, 2009.

[21] V. Keyantuo and C. Lizama, "A characterization of periodic solutions for time-fractional differential equations in $U M D$ spaces and applications," Mathematische Nachrichten, vol. 284, no. 4, pp. 494-506, 2011.

[22] M. Klimek, "Sequential fractional differential equations with Hadamard derivative," Communications in Nonlinear Science and Numerical Simulation, vol. 16, no. 12, pp. 4689-4697, 2011.

[23] Z. Wei, Q. Li, and J. Che, "Initial value problems for fractional differential equations involving Riemann-Liouville sequential fractional derivative," Journal of Mathematical Analysis and Applications, vol. 367, no. 1, pp. 260-272, 2010.

[24] K. Deimling, Multivalued Differential Equations, Walter de Gruyter, Berlin, Germany, 1992.

[25] S. Hu and N. S. Papageorgiou, Handbook of Multivalued Analysis, Theory I, Kluwer, Dordrecht, The Netherlands, 1997.

[26] A. Granas and J. Dugundji, Fixed Point Theory, Springer, New York, NY, USA, 2005.

[27] A. Lasota and Z. Opial, "An application of the Kakutani-KY Fan theorem in the theory of ordinary differential equations," Bulletin de l'Académie Polonaise des Sciences. Série des Sciences Mathématiques, Astronomiques, et Physiques, vol. 13, pp. 781786, 1965.

[28] A. Bressan and G. Colombo, "Extensions and selections of maps with decomposable values," Studia Mathematica, vol. 90, no. 1, pp. 69-86, 1988.

[29] M. Frigon, "Théorèmes d'existence de solutions d'inclusions différentielles," in Topological Methods in Differential Equations and Inclusions, A. Granas and M. Frigon, Eds., vol. 472 of NATO ASI Series C, pp. 51-87, Kluwer Academic Publisher, Dordrecht, Germany, 1995.

[30] H. Covitz and S. B. Nadler, "Multi-valued contraction mappings in generalized metric spaces," Israel Journal of Mathematics, vol. 8, pp. 5-11, 1970.

[31] M. Kisielewicz, Differential Inclusions and Optimal Control, Kluwer, Dordrecht, The Netherlands, 1991.

[32] C. Castaing and M. Valadier, Convex Analysis and Measurable Multifunctions, Lecture Notes in Mathematics, Vol. 580, Springer, Berlin, Germany, 1977. 


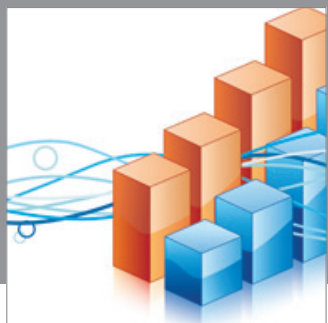

Advances in

Operations Research

mansans

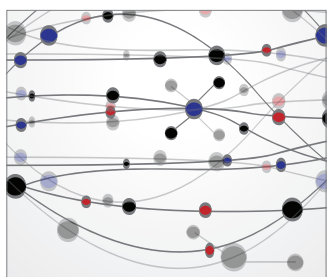

The Scientific World Journal
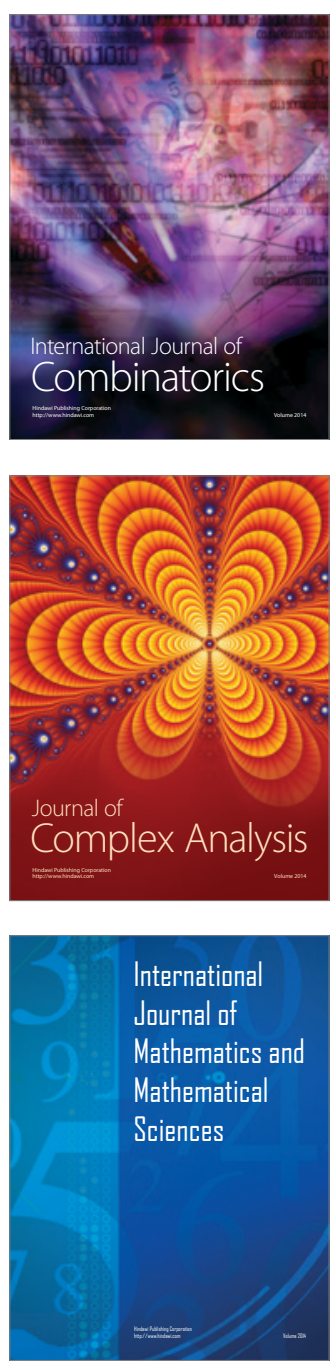
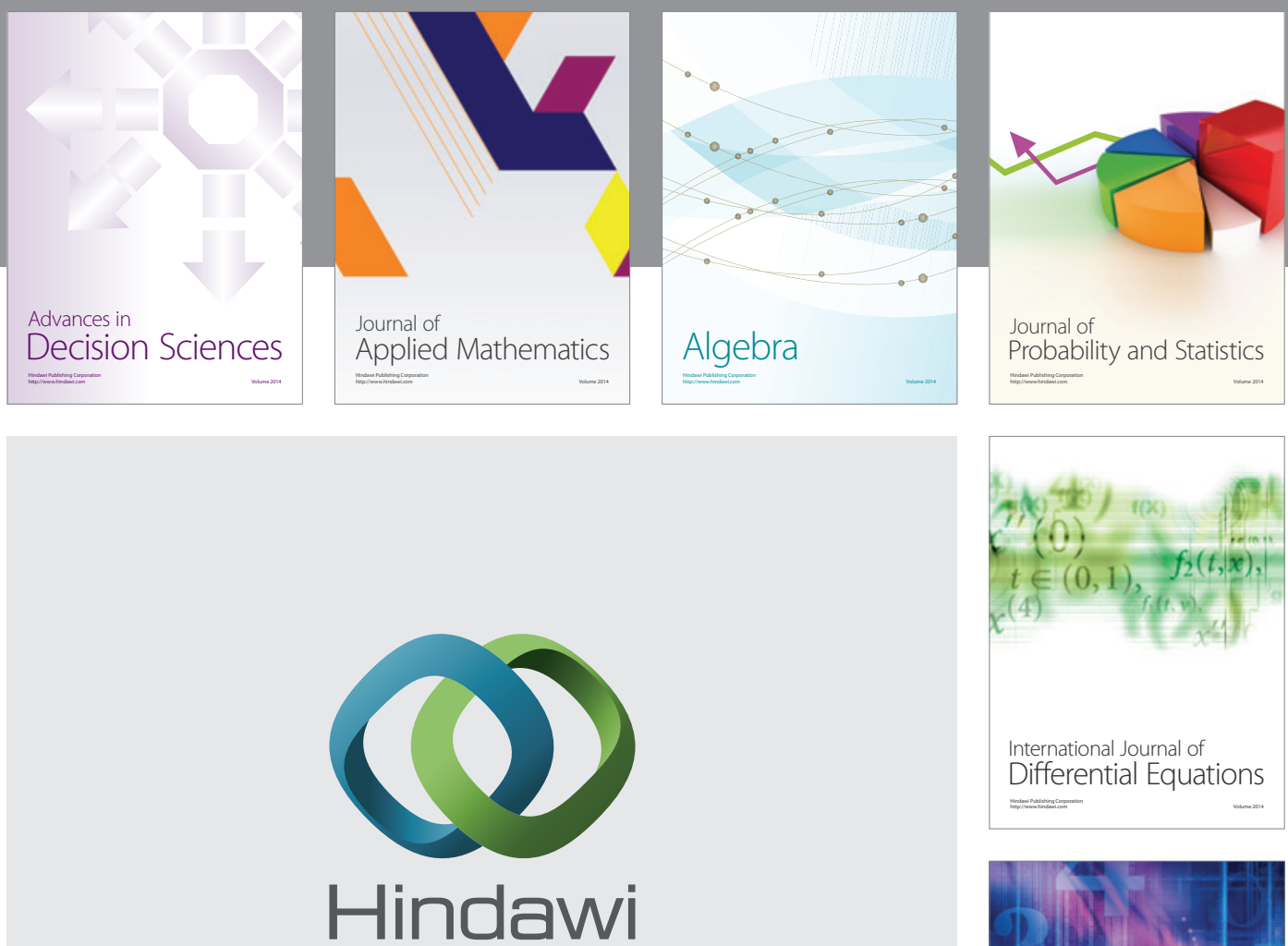

Submit your manuscripts at http://www.hindawi.com
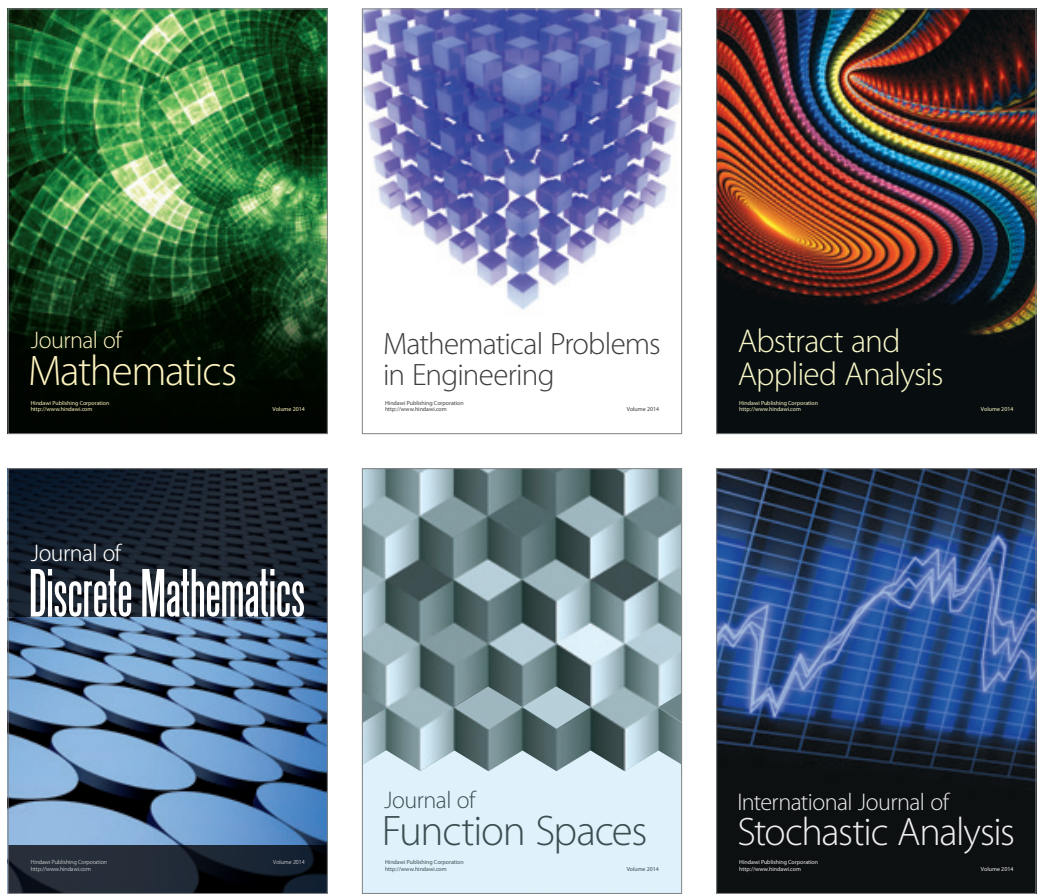

Journal of

Function Spaces

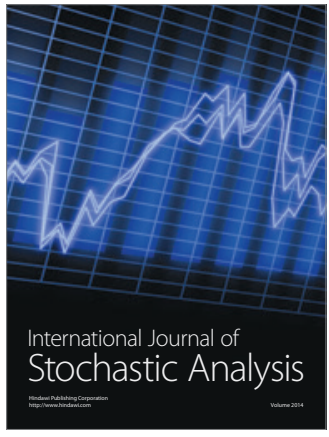

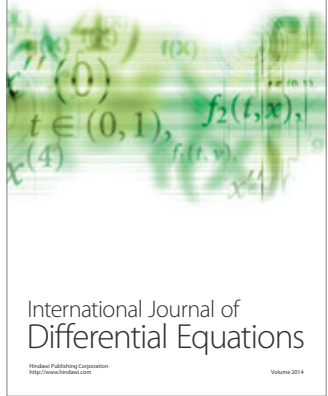
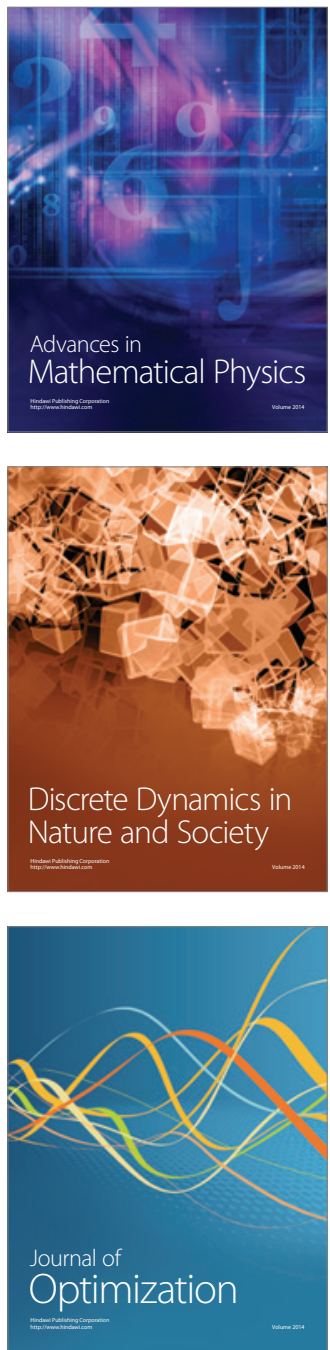